\title{
Efectos de la calidad del forraje, fresco 0 henificado, sobre el comportamiento alimentario del canero (ovis aries)
}

\author{
Carlos Álvarez Días'; Roberto Ruiz Pierruges² \\ Universidad Técnica de Machala' \\ Estación Experimental de Pastos y Forrajes de $\mathrm{Cuba}^{2}$ \\ caalvarez@utmachala.edu.ec
}

\section{Resumen}

Con el objetivo de estudiar la influencia de la calidad y forma física del forraje voluminoso sobre el comportamiento alimentario del carnero, seis machos adultos Pelibuey, 40-45 Kg, en jaulas de metabolismo fueron alimentados una vez al día con cuatro dietas, forraje verde, heno largo bueno y malo troceadas y heno malo molido, todas de pasto estrella (Cynodon mlenfuensis) y agua ad-libitum. En un diseño cuadrado latino incompleto con análisis de varianza simple y dócima de Duncan, dos animales permanecieron como control, heno malo troceado y cuatro rotaron 7 días por cada dieta con registró mecanográfico mandibular circadiano continuo las últimas 48 horas. Los resultados muestran menor tiempo de ingestión, rumia y ciclos mericicos con mayor tiempo de reposo y consumo de MS y menor ingestión de agua en la dieta de forraje verde. El heno molido tiene poco efecto sobre los tiempos de ingestión, rumia y reposo pero incrementa el consumo de MS y agua para esta forma de conservación del forraje. La duración de las grandes comidas disminuye con la calidad del forraje. El forraje verde y el heno malo molido no afectan los períodos de reposo. La Duración Diaria de Ingestión y de Rumia disminuye en el forraje verde mientras la Duración Unitaria de Ingestión presenta diferencias entre los forrajes y la de Rumia es superior para el heno malo largo. El esfuerzo masticatorio es superior para el heno largo. El Índice de Fibrosidad Diario de Rumia es superior para el heno largo bueno. Se discuten los resultados.

Palabras clave: Comportamiento alimentario, carneros, alimentos voluminosos, calidad, forma física.

\section{Abstract}

The objective was to study the effect of quality and form of voluminous diets on the feeding behavior of sheep. Six adult male Pelibuey (weigh 40-45kg) placed in metabolism cages where feeding with 4 different voluminous diets: green forage, good and bad large hay and short bad hay. In a latin square incomplete design, two control animals received a short bad hay ration to eliminate the environmental effects and the others rotated.by the different diets. Animals were feeding once a day $(9 \mathrm{am})$ and disposed water ad-libitum. Feeding behavior components, its circadian

Cómo citar este artículo: Álvarez C. y Ruiz R. (2015) Efectos de la calidad del forraje, fresco o henificado, sobre el comportamiento alimentario del canero (ovis aries). CUMBRES, Revista Científica. 1(2) 08 - 11

\section{$8 \quad$ Cumbres}


distribution and mastication effort were recorded by mandibular mecanograme during 48 hours.. Results evidence that green forage reduce the times of ingestion, rumination, mericique cicles and water consumption increasing rest time and DM ingestion. Lower effect produce short bad hay on the distribution of circadian feeding behavior but increase DM and water consumption. There is an inverse relation between duration of big food and quality of forage at the time that green forage and short bad hay doesn't affect resting times. Ingestion Dairy Duration and Rumination Dairy Duration diminish in green forage while Ingestion Unitary Duration present different between forages but Rumination Unitary Duration is higher in short bad hay. Mastication effort and Roughage Dairy Rumination Index (RDRI) increases in large good hay. Results are discussed.

Keywords: Animal behavior-sheep-voluminous dietseffect of quality and form

\section{Introducción}

Los rumiantes constituyen la forma más evolucionada de los herbívoros al presentar un sistema digestivo adaptado a consumir una dieta seca y fibrosa, de la cual pueden sobrevivir debido a las modificaciones digestivas y metabólicas producidas durante un largo proceso evolutivo ontogénico (primario) y por el mejoramiento zootécnico (contemporáneo) (Ruiz Pierruges, 2001, Álvarez Díaz, 2007).

Se estima que los forrajes, en todas sus formas de oferta, constituyen el 90\% de la energía del alimento consumida por los herbívoros a nivel mundial (Givens et al. 2000).

El comportamiento animal es la reacción del organismo como un todo ante determinados estímulos externos y/o internos, es decir, es la forma en que este reacciona (Broom, 1999) por lo que toda acción conductual es la manifestación externa de la satisfacción de una necesidad de vida (Álvarez Díaz y Dabesa, 2008). El comportamiento alimentario es una forma de la conducta en donde el factor animal tiene que ser estudiado en estrecha relación con los factores alimento, ambiente y humano (Álvarez Díaz et al, 2009).

\section{Materiales y métodos}

En un diseño de cuadrado latino incompleto con análisis de varianza simple y dócima de Duncan para conocer el grado de significación estadística, seis carneros machos y adul- tos de la raza Pelibuey, con peso promedio de 40-45 Kg. fueron alojados en jaulas individuales de metabolismo; dos animales permanecieron como controles con una dieta constante de heno molido para eliminar el efecto ambiente. Las dietas estudiadas fueron: forraje verde, heno largo bueno y heno largo malo troceados, y heno malo molido de pasto estrella (Cynodon mlenfuensis). La comida se ofertó en una sola ración al día (9:00 am); los animales dispusieron de agua fresca ab-libitum.

La técnica empleada fue el registro continuo circadiano del mecanograma mandibular mediante agujas inscriptoras de Marek en bandas negro de humo que garantizan gran fidelidad en el trazado para el estudio de la conducta alimentaria (Álvarez Díaz, 1986); para su lectura, las bandas negro de humo fueron fijadas con una solución alcohólica de goma laca y leídas en función a su velocidad de desplazamiento. Se estudiaron los indicadores de la distribución circadiana (24 horas) del comportamiento alimentario (ingestión, rumia y reposo), los niveles de ingestión de materia seca (MS) y agua así como las duraciones diarias de ingestión (DDI), de rumia (DDR) y de masticación (DDM). Los animales permanecieron 7 días en cada dieta con registro continuo las últimas 48 horas.

\section{Resultados y discusión}

La composición química del alimento, \% en base seca, muestra valores inferiores para los indicadores químicos determinados para el heno malo largo y molido mientras que los mejores valores nutritivos se correspondieron con el forraje verde y el heno largo bueno (Tabla 1).

Tabla 1. Composición química del alimento (\% en base seca)

\begin{tabular}{|l|c|c|c|c|c|c|c|}
\hline Alimento & MS & FB & PB & MOD $^{1}$ & EM $^{2}$ & PDIN $^{3}$ & PDIE $^{3}$ \\
\hline Forraje verde & 21.92 & 31,2 & 8,4 & 56,9 & 8,1 & 5,4 & 7,3 \\
\hline Heno largo bueno & 83,57 & 30,9 & 9,2 & 57,3 & 8,2 & 5,9 & 7,5 \\
\hline Heno largo malo & 85,99 & 41,6 & 5,7 & 42,8 & 5,9 & 3,3 & 4,8 \\
\hline Heno malo molido & 85,77 & 39,7 & 5,5 & 45,7 & 6,1 & 3,4 & 5,2 \\
\hline
\end{tabular}
Elaborado por: (Álvarez y col. 2014)

'Determinación in vitro

${ }^{2} \mathrm{MJ} / \mathrm{Kg}$ MS estimada a partir de MOD

3Estimados según García Trujillo t Cáceres (1984)

El tamaño de las partículas del alimento como se puede apreciar en la Tabla 2, para los alimentos voluminosos troceados forraje verde, heno bueno y malo, presentaron una distribución de estratificación muy parecida ya que del 92 al 93\% de sus partículas tenían un tamaño superior a 6,3 $\mathrm{mm}$ y del 4 al $8 \%$ eran mayores a 2,5 mm; el molinaje del heno permitió una importante reducción en la talla física de las partículas que se ubicaron entre 1,000 y 0,125 mm 
destacándose las partículas de 0,136 mm como las más abundantes (40\%).

Tabla 2. Porcentaje de distribución de las partículas del alimento según su estratificación de tamizado (diámetro del tamiz en mm)

\begin{tabular}{|c|c|c|c|c|c|c|c|}
\hline Alimento & 6,300 & 2,500 & 1,000 & 0,630 & 0,136 & 0,125 & $>0,125$ \\
\hline Forraje verde (FV) & 93,07 & 5,86 & 1,07 & 0 & 0 & 0 & 0 \\
\hline Heno largo bueno (HLB) & 92,42 & 4,92 & 1,33 & 0,66 & 0,47 & 0,18 & 0 \\
\hline Heno largo malo (HLM) & 93,14 & 4,06 & 1,56 & 0,39 & 0,50 & 0,19 & 0 \\
\hline Heno malo molido HMM) & 0,48 & 1,80 & 17,86 & 14,67 & 40,19 & 16,02 & 7,00 \\
\hline
\end{tabular}

La distribución circadiana de los componentes básicos de la conducta alimentaria (Tabla 3) muestra para el forraje verde un incremento significativo $(p<.001)$ en el tiempo de reposo debido principalmente a la reducción del tiempo de rumia $(p<.001)$ correspondiéndose con el valor más bajo de MS de las raciones estudiadas Es interesante destacar que las dietas de heno malo largo o molido produjeron los mayores tiempos de ingestión y rumia con independencia al tamaño de sus partículas lo que coincide con lo señalado por Mejía (2010) relativo a la influencia que tienen la calidad y el método de conservación del forraje sobre el nivel de consumo y esfuerzo de rumia puede explicarse, porque el forraje verde al tener menor contenido de fibra y lignina estimula la velocidad de pasaje o tránsito favoreciendo un mayor consumo en un período de tiempo más corto como reportan Dumont y Petit (1995). La limitación en la cantidad de alimento ingerida, bien en una comida o en un día, se debe a factores relacionados con el efecto de repleción del rumen tal como reportan Abazinge, Fontenot y Allen (1994) y Álvarez Díaz (1998).

Tabla 3. Distribución circadiana de los componentes del comportamiento alimentario (min)

\begin{tabular}{|l|c|c|c|c|}
\hline INDICADORES & FV & HLB & HLM & HMM \\
\hline INGESTION & $427^{\mathrm{a}}$ & $434^{\mathrm{a}}$ & $446^{\mathrm{a}}$ & $445^{\mathrm{a}}$ \\
\hline RUMIA & $396^{\mathrm{b}}$ & $515^{\mathrm{a}}$ & $558^{\mathrm{a}}$ & $529^{\mathrm{a}}$ \\
\hline REPOSO & $617^{\mathrm{b}}$ & $451^{\mathrm{a}}$ & $436^{\mathrm{a}}$ & $466^{\mathrm{a}}$ \\
\hline
\end{tabular}

Elaborado por: (Álvarez y col. 2014)

Superíndices diferentes $p<0.001$

En función al método de distribución de la comida, la ingestión primaria fue diurna, mientras que la rumia y el reposo fueron principalmente nocturnos a lo largo del ciclo circadiano lo que ratifica lo planteado por Vasilatos y Wangsness (1986) referente a la influencia del método sobre el accionar conductual de los animales. El efecto general sobre el indicador reposo circadiano coincide con lo señalado por Ruckebusch, Phaneuf y Dunlop (1994), Álvarez Díaz (1986) y Quincosa, Álvarez Díaz y Senra (2006) que asocian el aumento o la disminución del tiempo de reposo en función a la oscilación circadiana del tiempo de rumia que a su vez depende de la oscilación del tiempo de ingestión.

Tabla 4. Niveles y relación de la ingestión de MS y agua

\begin{tabular}{|l|c|c|c|c|}
\hline \multicolumn{1}{|c|}{ INDICADORES } & FV & HLB & HLM & HMM \\
\hline MS (g/d) & $1148,32 \pm$ & $882,51 \pm$ & $868,794 \pm$ & $1011,57 \pm$ \\
& $47,99^{\mathrm{a}}$ & $47,88^{\mathrm{b}}$ & $47,37^{\mathrm{b}}$ & $43,31^{\mathrm{a}}$ \\
\hline Agua (1/d) & $0,31 \pm 0,17^{\mathrm{b}}$ & $1,29 \pm 0,17^{\mathrm{a}}$ & $1,64 \pm 0,17^{\mathrm{a}}$ & $1,86 \pm 0,10^{\mathrm{a}}$ \\
\hline $\begin{array}{l}\text { Relación agua/MS } \\
\text { (1/Kg) }\end{array}$ & $0,58 \pm 0,15^{\mathrm{b}}$ & $1,45 \pm 0,15^{\mathrm{a}}$ & $2,02 \pm 0,16^{\mathrm{b}}$ & $1,80 \pm 0.09^{\mathrm{a}}$ \\
\hline
\end{tabular}

Superíndices diferentes $p<0.001$

En la dieta de forraje verde, el nivel de ingestión de MS (Tabla 4) alcanzó valores elevados como con la dieta de HMM siendo ambos significativamente superiores $(p<0.001)$ mientras que el volumen de agua ingerida fue menor por lo que le correspondió a esta dieta la menor relación de agua/MS ingerida ( $p<0.001)$. Estos resultados ratifican lo señalado por Theodorou y France (2000) y Alvarez Díaz et al (2009) respecto a que el volumen de agua ingerida es inversamente proporcional al nivel de hidratación del alimento.

Tabla 5. Duraciones diarias de ingestión (DDI) y rumia (DDR) y trabajo masticatorio (DDM) por tipo de dieta ( $\mathrm{min} / \mathrm{kg}$ de MS ingerida/24 hrs)

\begin{tabular}{|l|c|c|c|c|}
\hline INDICADORES & FV & HLB & HLM & HMM \\
\hline DDI & $372,16 \pm$ & $513,94 \pm$ & $539.48 \pm$ & $462,81 \pm$ \\
& $36,28^{\mathrm{b}}$ & $36,66^{\mathrm{a}}$ & $35,85^{\mathrm{a}}$ & $22,19^{\mathrm{a}}$ \\
\hline DDR & $341,18 \pm$ & $604,84 \pm$ & $626,46 \pm$ & $540,70 \pm$ \\
& $26,78^{\mathrm{b}}$ & $26,89^{\mathrm{a}}$ & $27.14^{\mathrm{a}}$ & $18,02^{\mathrm{c}}$ \\
\hline DDM & $713,53 \pm$ & $1143,54 \pm$ & 1157,30 & $986,40 \pm$ \\
& $37,95^{\mathrm{b}}$ & $8,10^{\mathrm{a}}$ & $\pm 38,44^{\mathrm{a}}$ & $25,52^{\mathrm{c}}$ \\
\hline
\end{tabular}
Elaborado por: (Alvarez y Col. 2014)

Superíndices diferentes $p<0.001$

La tabla 5 muestra la laboriosidad del proceso masticatorio (DDM) expresada en la duración diaria de masticación (DDM) y de rumia (DDR), significativamente menores para la dieta de forraje verde al ser una dieta de más fácil ingestión y menor esfuerzo de rumia; en las dietas de heno, el trabajo masticatorio es superior e inclusive el efecto molinaje prácticamente no influye sobre la laboriosidad del mismo (Grafico 1), resultados que concuerdan con lo expresado por Álvarez Díaz (2007).

Figura 1. Duraciones diarias de Ingestión, rumia y masticación

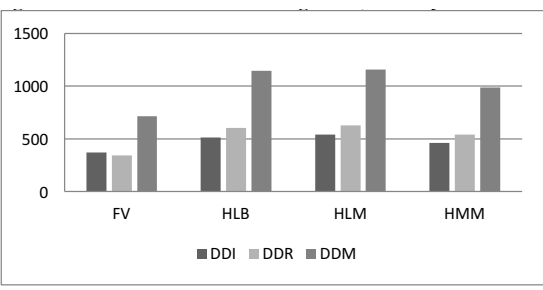

Elaborado por: (Álvarezy col. 2014)

\section{Cumbres}


Al comparar el esfuerzo masticatorio de rumia tomando como referencia el tiempo en minutos por gramo de MS y FB ingerida, Grafico 2, se aprecia que el tiempo empleado en la remasticación de la FB (Índice de Fibrosidad Diario de Rumia) muestra el menor tiempo para el forraje verde, el mayor para el heno largo bueno mientras que el heno malo, largo o molido, mostraron valores intermedios entre 1,5 y 1,9 min/g FB ingerida; estos resultados se explican, como expresa Álvarez Díaz (2007) por la característica física y grado de hidratación del alimento. La laboriosidad del trabajo de rumia para la FB es casi 2,5 veces superior al cálculo por gr de MS ingerida para todas las dietas.

Figura 2. Laboriosidad masticatoria de rumia

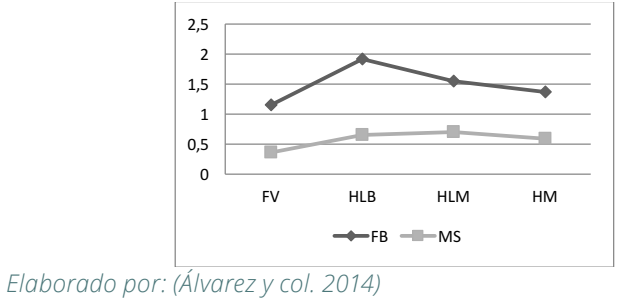

\section{Conclusiones}

1. La alimentación con forraje verde determinó una reducción de los tiempos de ingestión y rumia, con incremento del tiempo de reposo y consumo de MS y menor volumen de ingestión de agua.

2. El heno molido tiene poco efecto sobre los tiempos de ingestión, rumia y reposo pero incrementa el consumo de MS y agua para este tipo de conservación del forraje.

3. Las Duraciones Diarias de Ingestión (DDI) y de Rumia (DDR) disminuyen en el forraje verde mientras que la Duración Diaria de Masticación (DDM) se incrementa con el heno independientemente de su calidad y forma física lo que muestra el esfuerzo masticatorio para esta forma de conservación del forraje.

4. El cálculo para determinar el esfuerzo masticatorio es más objetivo en base a FB que en base a MS.

\section{Referencias bibliográficas}

- Abazinge, M.D.A., Fontenot, J.R. and Allen, V.G. (1994). Digestibility nitrogen utilization and voluntary intake of ensiled. J.anim.Sci.
- Álvarez Díaz. (1986). Efecto de la suplementación proteica y energética en el comportamiento alimentario del carnero. Tesis Doctor en Ciencia Veterinaria., ICA-UNAH. La Habana. Cuba.

- Álvarez Díaz. (1998). Fisiología del consumo voluntario y de la actividad ingestiva en los rumiantes. Monografía. Dpto. de Morfofisiología. Fac. Medicina Veterinaria. UNAH. La Habana. Cuba.

- Álvarez Díaz. (2007). Fisiología Digestiva Comparada de los Animales Domésticos. Imprenta Machala. El Oro. Ecuador.

- Álvarez Díaz; Dabesa, G. (2008). Behaviour and Welfare of Domestic Animals. Faculty of Veterinary Medicine. University of Gondar. Ethiopia.

- Álvarez Díaz, Pérez, H., De la Cruz, M., Quincosa, J., Pompa, A., Torres, E. (2009). Fisiología Animal Básica. Editorial F. Varela. MES. La Habana. Cuba.

- Bae, D., Welch, J., Smith, A. (1981). Efficiency of mastication in relation to hay intake by cattle. J. Anim.

- Broom, D. (1999). Animal welfare, the conceptand the issues. In: Dolins, F.L. (Editor). Attitudes to animals, views in animal welfare. Cambridge University Press. Cambrigde, U.K.

- Dumont, B., Petit, J. (1995). An indoor meted for studying the preferences of sheep an cattle at pasture.

- Givens, D., Owen, G., Axford, R., Omed, H.M. (2000). Forage evaluation in ruminanat nutrtion. CABI Publishing. UK

- Mejia, J. (2010). Consumo voluntario de forraje por rumiantes en pastoreo (II Parte). Factores que afectan el consumo voluntario. II Seminario Internacional de Agrostología y Manejo de Patos. Universidad de Guanajuato. Méjico. Disponible en Internet: www.agrytec.com/ pecuario/index.php?option=com_content\&id=1906

- Quincosa, J., Álvarez Díaz, Senra, A. (2006). Conducta ingestora de hembras bovinas en desarrollo del genotipo Siboney de Cuba en los periodos Iluvioso y poco lluvioso.

- Ruckebusch, Y., Phaneuf, L., Dunlop, R. (1994). Fisiología de pequeñas y grandes especies. Edit. Mundo Moderno. Méjico.

- Ruiz, R. (2001). Ciclo de actualización en ciencias agrarias. bovinocultura para la producción de leche. nutrición y agrostología. Instituto de Investigaciones de Pastos y Forrajes. Ministerio de la Agricultura, La Habana, Cuba

- Theodorou, M., France, J. (2000). Feeding systems and feed evaluation models. CABI Publishing. UK 